\title{
Scanning optical pyrometer for measuring temperatures in hollow cathodes
}

\author{
J. E. Polk and C. M. Marrese-Reading \\ Jet Propulsion Laboratory, California Institute of Technology, Pasadena, California 91109 \\ B. Thornber ${ }^{\text {a) }}$ \\ International Space University, 67000 Strasbourg, France \\ L. Dang ${ }^{\text {b) }}$ \\ California Institute of Technology, Pasadena, California 91109 \\ L. K. Johnson and I. Katz \\ Jet Propulsion Laboratory, California Institute of Technology, Pasadena, California 91109
}

(Received 4 May 2007; accepted 31 July 2007; published online 10 September 2007)

\begin{abstract}
Life-limiting processes in hollow cathodes are determined largely by the temperature of the electron emitter. To support cathode life assessment, a noncontact temperature measurement technique which employs a stepper motor-driven fiber optic probe was developed. The probe is driven inside the hollow cathode and collects light radiated by the hot interior surface of the emitter. Ratio pyrometry is used to determine the axial temperature profile. Thermocouples on the orifice plate provide measurements of the external temperature during cathode operation and are used to calibrate the pyrometer system in situ with a small oven enclosing the externally heated cathode. The diagnostic method and initial measurements of the temperature distribution in a hollow cathode are discussed. () 2007 American Institute of Physics. [DOI: 10.1063/1.2774828]
\end{abstract}

\section{INTRODUCTION}

Hollow cathodes used as thermionic electron sources in ion thrusters have demonstrated lifetimes of over $16000 \mathrm{~h}$ in space and over $30000 \mathrm{~h}$ in ground tests, ${ }^{1-4}$ but certain proposed future NASA missions have even more demanding life requirements. The temperature is the primary driver for the chemical processes and physical transport phenomena associated with cathode failure modes and a key indicator of cathode health, but has not been well characterized. Lack of knowledge about the internal temperatures results in large uncertainties in the achievable lifetime of hollow cathodes. To provide data for hollow cathode life models, we have developed the capability to make accurate internal temperature measurements using a fast scanning fiber optic probe and a ratio pyrometer system.

The electron emitter of a state-of-the-art hollow cathode is a porous tungsten tube (the "insert") with a bariumcontaining compound in the pores. The insert is mounted in a refractory metal tube with a plate containing a small orifice welded to the downstream end, as shown in Fig. 1. Contact temperature measurements on the interior are difficult because of the plasma environment and because they disturb the surface chemistry and electron emission processes. Optical access to the interior surface of the emitter is limited, making remote pyrometric measurements difficult. Most estimates of emitter temperature are based on thermocouple measurements of the exterior temperature. ${ }^{5-7}$ The tempera-

\footnotetext{
${ }^{a)}$ Present address: Department of Aerospace Sciences, Cranfield University, United Kingdom.

${ }^{b)}$ Present address: 1432 Kwis Ave., Hacienda Heights, CA 91745.
}

ture difference between these locations and the actual emitting surface is not known, however, and depends on the operating conditions and cathode thermal design. The lifelimiting processes are exponentially dependent on the emitter temperature, so uncertainties in temperatures inferred from measurements on the exterior limit their utility in assessing cathode life.

Several attempts to characterize cathode emitter temperatures have been made. Siegfried and Wilbur constructed a cathode which had an emitter tube made from a $0.025 \mathrm{~mm}$ thick tantalum foil coated on the inside with R-500, a barium-containing compound which maintained a low work function surface for a limited period of time. ${ }^{8}$ The tantalum cathode was contained within a quartz tube, allowing the temperature on the outside of the cylindrical foil emitter to be measured with a micro-optical pyrometer. The emitter surface temperature was assumed to be the same as the external temperature, which was justified in this case because of the use of very thin foil. This approach enables good optical access to the insert, but the geometry does not allow the use of the materials and processes that are important in impregnated porous tungsten inserts or the thermal environment of a conventional cathode assembly to be reproduced.

Salhi et al. ${ }^{5}$ and Salhi ${ }^{9}$ performed limited optical measurements of the internal temperature distribution on an impregnated insert in a xenon hollow cathode using a quartz fiber optic probe. The fiber probe was coated with a ceramic to protect it from the plasma, and the probe end was cut at a $45^{\circ}$ angle and coated with tantalum. This reflective coating acted as a mirror, directing light from a small hole in the ceramic coating on the side into the fiber. The probe was 


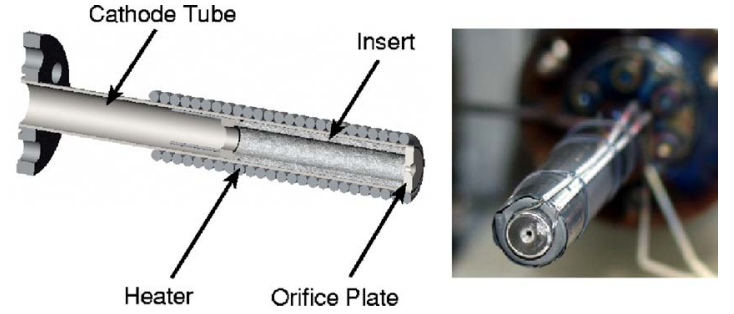

FIG. 1. Cutaway diagram showing the cathode configuration and photograph of the cathode with two thermocouples installed on the edge of the orifice plate.

inserted and removed rapidly by hand before it melted, and light collected by the fiber as it scanned axially along the insert was measured with a $\mathrm{SiGe}$ sandwich detector sensitive to wavelengths between $400-1000$ and $1000-1800 \mathrm{~nm}$. This was a novel approach to gaining optical access, but suffered from several limitations. The geometry of the probe tip resulted in collection of light from a relatively large area, limiting the spatial resolution. Contributions of plasma radiation to the collected signal were significant. Finally, the system was calibrated using a tungsten ribbon lamp, and no consideration was given to the effect of reflected light in the hollow cathode cavity or surface chemistry of the insert on the effective surface emittance.

The apparatus described in this article is designed to address these issues and provide reliable internal temperature measurements. Issues associated with plasma heating were avoided by using a high temperature sapphire fiber optic probe and a fast linear positioning system. Feedback from a rotary encoder on the actuator provides a position measurement resolution of $0.031 \mathrm{~mm}$, while eliminating reflective coatings and relying on total internal reflection in the fiber yield a spatial resolution of about $2 \mathrm{~mm}$. An optical system employing narrow bandpass interference filters eliminates plasma line radiation. Measurements at two discrete wavelengths allows the use of ratio pyrometry to minimize sensitivity to variations in the distance of the probe to the surface. Finally, the system is calibrated in situ using the hollow cathode heater and thermocouples on the orifice plate, which automatically compensates to a large extent for cavity and surface effects on the effective emittance. This article describes the temperature measurement system and presents initial measurements made on a hollow cathode over a range of operating conditions.

\section{FAST SCANNING PYROMETER SYSTEM}

The system is designed to fit the constraints of the hollow cathode geometry and mounting system and consists of the fiber optic probe, the motion control system, and the pyrometer.

\section{A. Test article and support equipment}

The cathode used in these experiments is shown in Fig. 1. The porous tungsten insert is impregnated with barium oxide, calcium oxide, and alumina in the molar ratios used in most conventional dispenser cathodes. ${ }^{10,11} \mathrm{~A}$ molybdenum ring and three equally spaced current leads are brazed

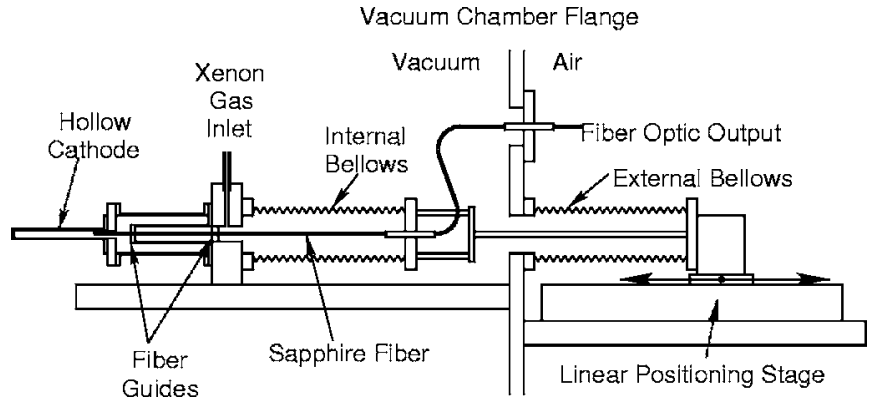

FIG. 2. Schematic of the fiber optic probe motion system.

to the upstream end of the insert. A swaged coaxial heater wrapped around the downstream end of the cathode is used to condition the emitter and to preheat it prior to ignition. Tantalum radiation shielding is wrapped around the heater coil to improve the heater efficiency. Two thermocouples are spot welded to the edge of the orifice plate, as shown in Fig. 1. Fine thermocouple wire is used to minimize heat conduction from the orifice plate and the junctions are covered with nickel foil that serves as a radiation shield. A flat molybdenum plate is used as the anode. In these tests no intermediate keeper electrode or applied magnetic field was used.

The cathode assembly is mounted in a $1 \mathrm{~m}$ diameter by $2 \mathrm{~m}$ long vacuum facility pumped by two $25 \mathrm{~cm}$ diameter cryopumps. Pressure is monitored with an ion gauge calibrated with xenon gas. The base pressure is typically 4 $\times 10^{-5} \mathrm{~Pa}\left(3 \times 10^{-7}\right.$ Torr $)$ and the pressure during cathode operation ranges from $1.33 \times 10^{-2}$ to 2.66 $\times 10^{-2} \mathrm{~Pa}\left(1 \times 10^{-4}\right.$ to $2 \times 10^{-4}$ Torr $)$. Ultrahigh purity xenon is used as the cathode expellant. The flow rate is controlled with a thermal mass flow meter and solenoid valve. The meter is calibrated by flowing xenon into a known volume and measuring the rate of pressure rise with a precision pressure transducer, yielding flow rate measurements with an uncertainty of less than $2 \%$. An Optomux data system with LABVIEW control software is used for flow setpoint control and flow meter data logging. Heater and discharge power are provided by laboratory power supplies. The cathode is electrically grounded to the chamber through the mounting structure. Currents and voltages are measured to within $1 \%$ by the data system using calibrated shunts and voltage dividers.

\section{B. Insert temperature probe}

Light from the interior surface of the insert is collected with a $425 \mu \mathrm{m}$ diameter sapphire fiber. The end of the fiber is cut and polished at a $45^{\circ}$ angle to the fiber axis, which rotates the fiber acceptance cone by $90^{\circ}$. The fiber field of view is therefore sideways, and it collects light from a region about $2 \mathrm{~mm}$ in axial extent and $180^{\circ}$ azimuthally. As shown schematically in Fig. 2, the probe is located inside a gas plenum upstream of the cathode. The fiber is terminated with a standard SubMinature version A connector and is attached to a fiber optic feedthrough at the upstream end of a bellows, which allows probe motion parallel to the axis of the cathode. The probe is carefully positioned to scan along the interior of the cathode approximately $0.5-1 \mathrm{~mm}$ from the insert surface. It is normally parked upstream of the cathode 


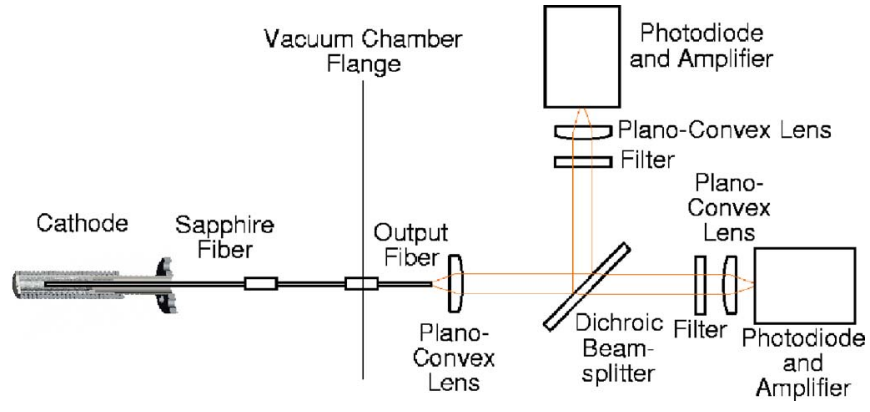

FIG. 3. Schematic of the two-color pyrometer system.

and is driven downstream by the positioning system a total distance of $52 \mathrm{~mm}$, which brings the tip within $1.7 \mathrm{~mm}$ of the upstream surface of the orifice plate.

\section{Motion control system}

The probe is moved with a fast, stepper motor-driven positioning system located outside the chamber. Another bellows feedthrough on the chamber forms the vacuum seal, as shown in Fig. 2. The peak speed of the linear positioning system is $350 \mathrm{~mm} / \mathrm{s}$, and with a programed acceleration and deceleration profile at the start and end points, the probe travels in and out of the cathode tube and insert in about $400 \mathrm{~ms}$. The probe location is monitored with $0.031 \mathrm{~mm}$ resolution using a rotary encoder. Lateral motion of the fiber is prevented using two fiber guides upstream of the cathode. A flexible fiber optic cable transmits the light signal from the movable stage inside the vacuum chamber to a fiber optic feedthrough on the chamber door.

\section{Ratio pyrometer system}

The signal is transmitted from the chamber to the optics system shown in Fig. 3 with another flexible fiber optic cable. Light exiting the fiber is collimated onto a long wave pass dichroic beam splitter. The beam splitter transmits wavelengths around $1500 \mathrm{~nm}$ with an efficiency of $85 \%$ and reflects shorter wavelengths around $1260 \mathrm{~nm}$ with $97 \%$ efficiency. The reflected and transmitted beams are filtered using interference filters with a $10 \mathrm{~nm}$ bandpass centered on 1200 and $1500 \mathrm{~nm}$, then focused onto Hammamatsu InGaAs photodiodes. These wavelengths were chosen to give good spectral separation for ratio pyrometry and because there is no interfering xenon line radiation. We assume that minor components of the plasma such as barium and barium oxide do not contribute significantly to the plasma radiation.

The voltage signals from amplifiers in the photodiode assemblies are collected with a data acquisition system. The output from the rotary encoder on the positioning system is used to time the data acquisition, so the photodiode signals are perfectly coordinated with the motion. The voltage output from photodiode $i$ is given by the expression

$$
\begin{aligned}
V_{\text {out }, i}= & V_{0, i} \\
& +G_{t, i} R_{i}\left(\lambda_{i}\right) \tau_{o}\left(\lambda_{i}\right) \tau_{f}\left(\lambda_{i}\right) \epsilon_{a}\left(\lambda_{i}\right) F_{g} \Delta \lambda_{f, i} L_{\lambda, b}\left(\lambda_{i}, T\right),
\end{aligned}
$$

where $V_{0, i}$ is the voltage offset of amplifier $i$ (signal with no illumination), $G_{t, i}$ is the transimpedence gain of the internal amplifier in V/A, $R_{i}$ is the responsivity of the detector in $\mathrm{A} / \mathrm{W}, \tau_{o}$ is the transmission of the optics system, $\tau_{f}$ is the fiber transmission, and $\Delta \lambda_{f, i}$ is the bandpass of interference filter $i$, which is centered on the wavelength $\lambda_{i}$. The apparent surface emittance $\epsilon_{a}\left(\lambda_{i}\right)$ depends on the true emittance of the surface in the field of view as well as the temperature distribution and optical characteristics of the cathode interior. $F_{g}$ is a geometric factor which is a function of the distance of the fiber from the surface and the acceptance angle, but not the wavelength. The spectral radiance in $\mathrm{W} / \mathrm{m}^{2} \mathrm{sr}$, $L_{\lambda, b}\left(\lambda_{i}, T\right)$, is approximated well in this wavelength and temperature range by the Wien formula, ${ }^{12}$

$$
L_{\lambda, b}\left(\lambda_{i}, T\right)=\frac{c_{1}}{\lambda_{i}^{5}} \exp \left(-c_{2} / \lambda_{i} T\right),
$$

where $T$ is the surface temperature and $c_{1}$ and $c_{2}$ are constants. The net signal due to radiation from the insert surface can be expressed as

$$
V_{i}=V_{\text {out }, i}-V_{0, i}=\epsilon_{a} A_{i} F_{g} \exp \left(-B_{i} / T\right),
$$

where $A_{i}=G_{t, i} R_{i} \tau_{o} \tau_{f} \Delta \lambda_{f, i} c_{1} / \lambda_{i}^{5}$ and $B_{i}=c_{2} / \lambda_{i} . B_{1}$ and $B_{2}$ for the wavelengths used in this device depend only on physical constants and the two wavelengths. For the chosen wavelengths, $B_{1}(1500 \mathrm{~nm})$ is $9593 \mathrm{~K}$ and $B_{2}(1200 \mathrm{~nm})$ is $11991 \mathrm{~K}$. The ratio of the diode voltages is

$$
\frac{V_{1}}{V_{2}}=\frac{\epsilon_{a}\left(\lambda_{1}\right)}{\epsilon_{a}\left(\lambda_{2}\right)} \frac{A_{1}}{A_{2}} \exp \left[\frac{B_{2}-B_{1}}{T}\right] \text {. }
$$

The quantity $B_{2}-B_{1}$ is $2398 \mathrm{~K}$. With a suitable calibration, the system can be used as a single wavelength or ratio pyrometer. The primary advantage of the ratio technique is that the geometric factor $F_{g}$ cancels out, so the voltage ratio is insensitive to variations in the fiber-to-surface distance.

\section{CALIBRATION AND DATA ANALYSIS}

\section{A. Calibration method}

The function sought in the calibration relates the ratio of photodiode voltages to the surface temperature in the field of view of the fiber optic probe over a temperature range that encompasses that expected in the cathode. In this experiment the pyrometer response is calibrated in situ using the cathode heater itself. A small cylindrical cup consisting of tantalum radiation shields can be slipped over the cathode using a motion feedthrough in the vacuum chamber door. This en-

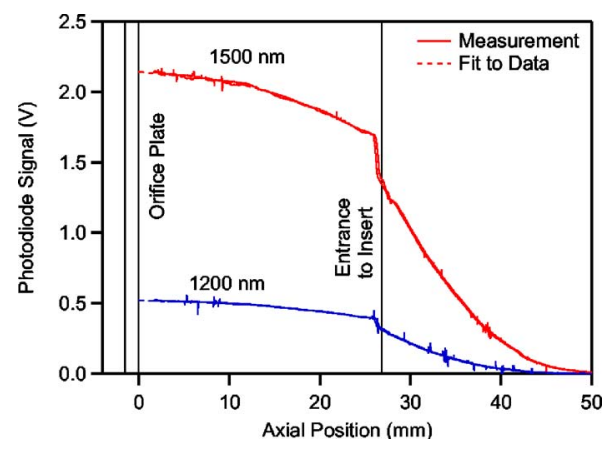

FIG. 4. Typical photodiode voltage signals with the calibration oven. 


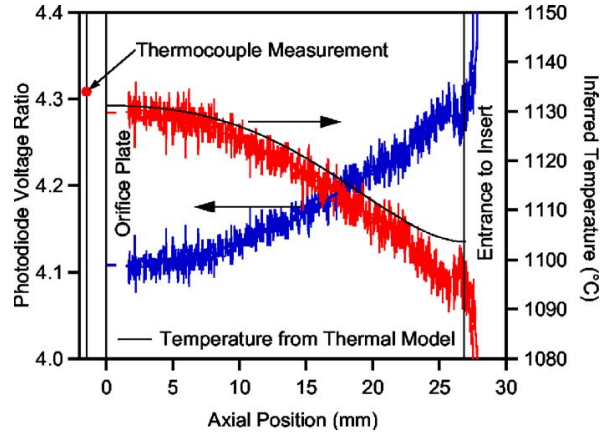

FIG. 5. Ratio of photodiode voltages with the calibration oven (red curve), temperatures inferred from the ratio curve (blue curve), and the temperature profile from the thermal model (black line).

closure and the cathode heater form an oven that provides a nearly isothermal region at the downstream end of the cathode, so the pyrometer signal there can be related to the temperatures measured with the thermocouples on the orifice plate. Figure 4 shows an example of the two photodiode signals. There is a large gradient upstream of the insert due to axial conduction and radiation losses from the unshielded portion of the cathode tube. Inside the oven, however, the gradient is much smaller and the signals are almost constant for the last $8 \mathrm{~mm}$ at the downstream end (i.e., from 2 to $10 \mathrm{~mm}$ ). The methods discussed in the next section can be used to show that the apparent emittance at the downstream end is essentially unity because the cavity is nearly isothermal. Figure 5 shows the ratio of photodiode voltages from Fig. 4 in the insert region. Similar curves were obtained for a range of heater powers.

The photodiode voltages were extrapolated to the orifice plate to obtain the peak photodiode signals, which are plotted as a function of the orifice plate temperature measured with the thermocouples in Fig. 6. The two thermocouples typically agree within $2{ }^{\circ} \mathrm{C}$ when the calibration oven is in place. The photodiode signals are very repeatable from scan to scan, but exhibit a slow monotonic decay over many scans, as shown in Fig. 6. No degradation was observed when the probe was parked in the upstream position; only as a result of scanning in the insert region. Examination of used fibers with energy dispersive spectroscopy in a scanning electron microscope revealed barium on the surface. This coating, which comes from barium vapor in the insert

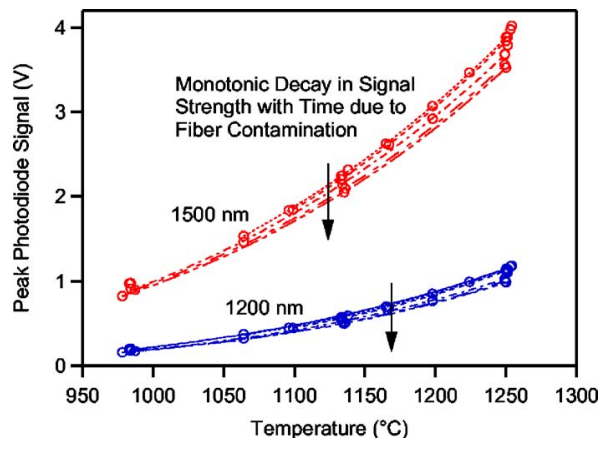

FIG. 6. Variation in peak photodiode voltages with temperature. Slow decay in signal with time is caused by contamination of the fiber optic probe with barium and barium-containing compounds.

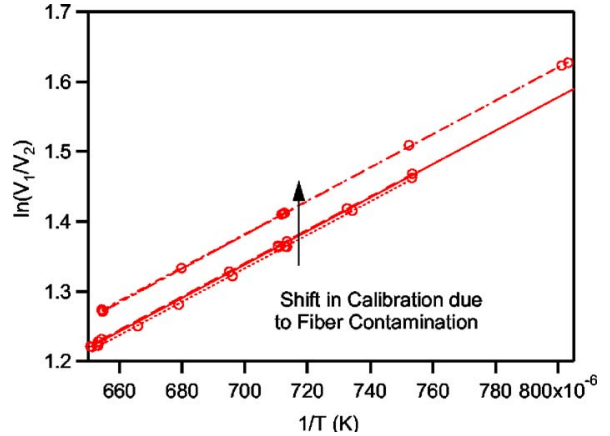

FIG. 7. Typical calibration curves. Shifts in calibration are due to probe contamination.

plasma, slowly degrades the fiber transmission. Calibrations must therefore be performed frequently to monitor the drift in response.

Equation (4) suggests that these data should follow the relationship $\ln \left(V_{1} / V_{2}\right)=\ln \left[\epsilon_{a}\left(\lambda_{1}\right) A_{1} / \epsilon_{a}\left(\lambda_{2}\right) A_{2}\right]+\left(B_{2}-B_{1}\right) / T$. When the data are plotted as $\ln \left(V_{1} / V_{2}\right)$ vs $1 / T$, we obtain the expected linear relationship, as shown in Fig. 7. The slope varies from 2490 to $2606 \mathrm{~K}$, however, which is $4 \%-8 \%$ higher than the theoretical value for $B_{2}-B_{1}$. Thermal modeling suggests that this discrepancy is due to small differences in the temperature of the orifice plate and the downstream end of the insert. The thermal characteristics of the operating cathode are difficult to model because of the complex heat inputs from the arc, but the known heat flux distribution from the heater allows the calibration geometry to be modeled with reasonable accuracy. A thermal model of the cathode assembly developed with COMSOL's MULTIPHYSICS simulation software incorporates a realistic geometry, temperaturedependent material properties, and conduction as well as radiation heat transfer. Contact resistances between the heater and the cathode tube and between the insert, tube, and the orifice plate were benchmarked using data over a range of heater power inputs from a similar cathode instrumented with eight thermocouples. Using measured values of heater power and with reasonable assumptions for the cathode mounting flange temperature and the temperature difference between the cathode assembly and the interior surface of the oven radiation shields, the orifice plate temperature and insert temperature profiles can be reproduced. Simulations over a range of heater powers yielded the temperature differences shown in Fig. 8. For most of the heater power range,

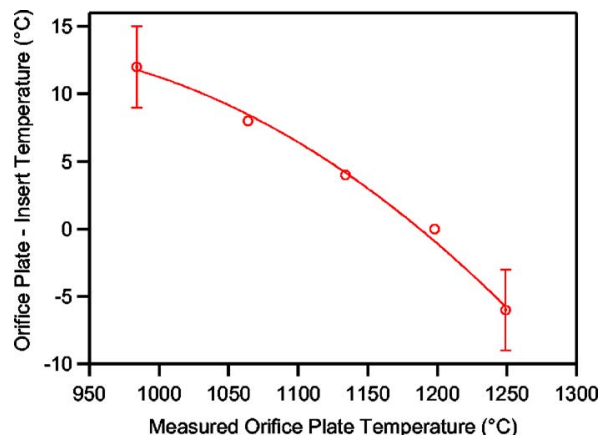

FIG. 8. Small differences in the temperature measured on the orifice plate and the actual peak temperature of the insert. 
the orifice plate is slightly hotter than the insert. At the highest heater powers, however, a peak heat flux in the center of the heater coil and radiation from the orifice plate result in orifice plate temperatures lower than the insert temperature. The error bars represent an uncertainty of $\pm 3{ }^{\circ} \mathrm{C}$, based on sensitivity tests with the thermal model.

Seven calibration curves measured while taking the data presented in the next section are shown in Fig. 7. The calibration temperatures used in these curves have been corrected to reflect the differences shown in Fig. 8, yielding constant slopes within $0.6 \%$ of the theoretical value. The inferred temperature for the voltage ratio in Fig. 5 is shown on the same plot. The insert temperature profile calculated with the thermal model for this heater input power is also plotted, demonstrating good agreement with the measured profile.

The decay in signal strength due to barium contamination causes a shift in the calibration, as shown in Fig. 7. The changes are gradual, with the exception of one episodic shift associated with operation at low flow which caused a transition to a higher discharge voltage and then discharge extinction. The small changes in the calibration due to barium deposition yield differences in the calculated temperature of only $2-3{ }^{\circ} \mathrm{C}$. The large shift observed in Fig. 7 results in calculated temperature differences of up to $30^{\circ} \mathrm{C}$ if applied to data taken before the shift. However, three operating points that were repeated after the major shift agreed with earlier measurements to within $2-5{ }^{\circ} \mathrm{C}$ when the proper calibration was used, demonstrating that the technique is reliable if calibrations are performed often.

\section{B. Apparent emittance correction}

The apparent emittance depends on the true emittance of the surface, internal radiation transport in the cavity, and the temperature distribution in the cavity. As noted above, the apparent emittance is approximately 1 under the calibration conditions, but during operation, larger thermal gradients on the insert and differences between the orifice plate and insert temperatures produce small changes to the apparent emittance, which must be corrected. This section describes an iterative correction method which uses a model of internal radiation, the true emittance of the interior surfaces, and measured temperature distributions.

The true emittance of tungsten depends on the temperature, the surface roughness, and surface chemical state. Measurements of the normal spectral emittance of tungsten with a surface roughness of $10-28 \mu \mathrm{m}$ rms at temperatures between 500 and $1380 \mathrm{~K}$ yield values ranging from 0.52 to 0.67 at $1200 \mathrm{~nm}$ and from 0.45 to 0.6 at $1500 \mathrm{~nm} .{ }^{13}$ For surfaces with $4-5 \mu \mathrm{m}$ rms roughness the values drop to as low as 0.45 and 0.38 at 1200 and $1500 \mathrm{~nm} .{ }^{13}$ Normal emittance values of 0.57 at $1200 \mathrm{~nm}$ and 0.47 at $1500 \mathrm{~nm}$ were measured on impregnated porous tungsten dispenser cathodes at room temperature by Simmons et al. ${ }^{14}$ At a temperature of $1111^{\circ} \mathrm{C}$, they estimated emittances of 0.51 and 0.48 at 1000 and $1800 \mathrm{~nm}$ based on a balance between input and radiated power for a very large dispenser cathode. In a very careful study, Jaafari and Free measured the emittance at $660 \mathrm{~nm}$ of a dispenser cathode operated at $1210{ }^{\circ} \mathrm{C}$ for over
$4000 \mathrm{~h}$ with two cold starts and one brief exposure to atmosphere. ${ }^{15}$ In the first $70 \mathrm{~h}$ the emittance increased from 0.45 to $0.46-0.47$ and then increased slowly to 0.48 at the end of the test. These results indicate that the emittance does not change dramatically due to the initial activation (when the chemical state of the surface is changing) or due to brief air exposures. Furthermore, the emittance is stable over long periods of time. We therefore assume that a single value of the surface emittance at each wavelength can be used to calculate the apparent emittance for the nonoperating cathode during calibration and at all conditions when the cathode is operating. For the measurements presented below, nominal values of 0.53 at $1200 \mathrm{~nm}$ and 0.48 at $1500 \mathrm{~nm}$ were chosen. The ranges noted above were used to bound the error due to emittance uncertainty in the next section.

The radiation incident on the tip of the fiber optic probe from a surface element in its field of view is the sum of radiation emitted from that surface and radiation from other parts of the cavity reflected from that surface. The total radiosity at a given wavelength and temperature $J\left(\lambda_{i}, T\right)$ can be written as ${ }^{12}$

$$
\begin{aligned}
J\left(\lambda_{i}, T\right) & =\epsilon_{a}\left(\lambda_{i}\right) L_{\lambda, b}\left(\lambda_{i}, T\right)=\epsilon\left(\lambda_{i}\right) L_{\lambda, b}\left(\lambda_{i}, T\right)+\rho_{i} G\left(\lambda_{i}, T\right) \\
& =\epsilon\left(\lambda_{i}\right) L_{\lambda, b}\left(\lambda_{i}, T\right)+\left(1-\epsilon\left(\lambda_{i}\right)\right) G\left(\lambda_{i}, T\right),
\end{aligned}
$$

where the first expression is the form used in Eq. (1). The second expression includes a term for the emitted radiation using the true emittance $\epsilon\left(\lambda_{i}\right)$ and a term for the reflected radiation, which depends on the irradiance $G\left(\lambda_{i}, T\right)$ from other parts of the cavity and the reflectance $\rho_{i}$ of the surface in view. The final expression uses the assumption that the surface does not transmit radiation, so the sum of the true emittance and reflectance is unity.

The correction algorithm uses a discretized form of this expression,

$$
J_{j}\left(\lambda_{i}, T_{j}\right)=\epsilon_{j}\left(\lambda_{i}\right) L_{\lambda, b}\left(\lambda_{i}, T_{j}\right)+\left(1-\epsilon_{j}\left(\lambda_{i}\right)\right) \sum_{k} F_{k j} J_{k}\left(\lambda_{i}, T_{k}\right),
$$

where subscript $j$ denotes the surface in view, $k$ denotes other discrete surfaces in the cavity, and $F_{k j}$ is the view factor between surfaces $k$ and $j$. This equation can be solved to give an explicit expression for the radiosity of a given surface $j$,

$$
\begin{aligned}
J_{j}\left(\lambda_{i}, T_{j}\right)= & \frac{1}{1-F_{j j}\left(1-\epsilon_{j}\left(\lambda_{i}\right)\right)}\left[\left(1-\epsilon_{j}\left(\lambda_{i}\right)\right) \sum_{k \neq j} F_{k j} J_{k}\left(T_{k}, \lambda_{i}\right)\right. \\
& \left.+\epsilon_{j}\left(\lambda_{i}\right) L_{\lambda, b}\left(T_{j}, \lambda_{i}\right)\right] .
\end{aligned}
$$

The cathode interior is divided into 86 surfaces composed of disk-, washer-, or ring-shaped elements, each with a characteristic length of $1 \mathrm{~mm}$ or less. The view factors between all pairs of surfaces are calculated with corrections for partial or complete shadowing by intervening surfaces. An initial estimate of the temperature distribution is obtained by assuming an emittance of 1 at each wavelength. The radiosities of all interior surfaces are calculated using this temperature distribution and the true emittances. An improved estimate of the effective emittance of each surface element is given by 


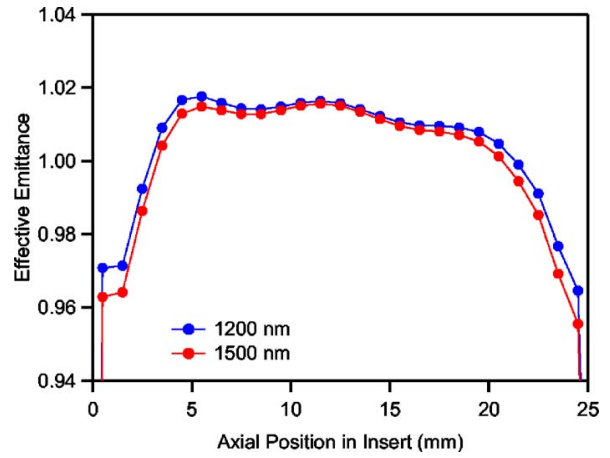

FIG. 9. Apparent emittance distribution in the hollow cathode cavity for a typical temperature distribution in an operating cathode.

$$
\epsilon_{a, j}\left(\lambda_{i}\right)=J_{j}\left(T_{j}, \lambda_{i}\right) / L_{\lambda, b}\left(\lambda_{i}, T_{j}\right) .
$$

The temperature distribution is then updated using the new emittances according to the expression

$$
\frac{1}{T_{j}\left(\epsilon_{a} \neq 1\right)}=\frac{1}{T_{j}\left(\epsilon_{a}=1\right)}-\frac{1}{\left(B_{2}-B_{1}\right)}\left[\ln \left(\frac{\epsilon_{a, j}\left(\lambda_{1}\right)}{\epsilon_{a, j}\left(\lambda_{2}\right)}\right)\right] .
$$

This process is repeated until the maximum temperature change from the previous iteration is less than $1{ }^{\circ} \mathrm{C}$, which typically required only three to four iterations. Figure 9 shows typical results for an operating cathode. Reflected radiation from cooler upstream portions of the insert reduces the effective emittance at the cathode tip. In the center of the insert, radiation from the hot downstream end results in an apparent emittance greater than 1. The apparent emittance of the upstream end is less than unity because of radiation from the cooler cathode tube. The apparent emittances at the two wavelengths are not very different, however. The effect of various assumptions for the true emittances on the apparent emittance ratio at the downstream end of the cathode and the peak temperature for a range of temperatures is shown in Fig. 10. The correction to the temperature using the nominal values for the emittances is only $3-4{ }^{\circ} \mathrm{C}$.

\section{Uncertainty analysis}

Random errors due to noise in the photodiode signals are reduced to negligible levels by averaging, as shown by the

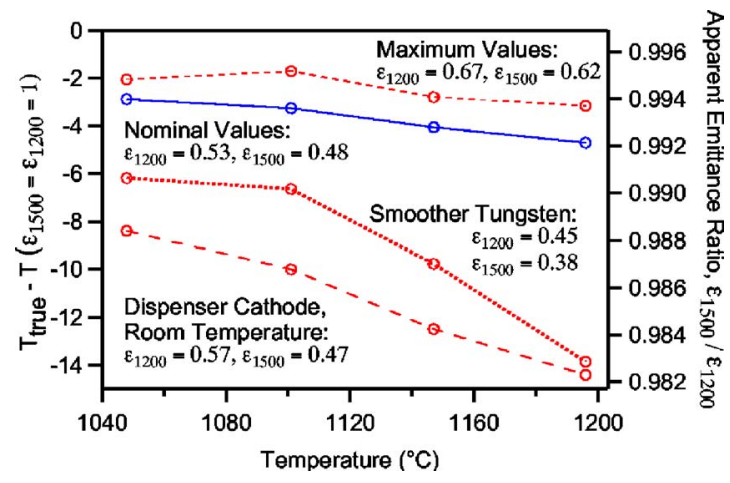

FIG. 10. Apparent emittance ratio at the downstream end of the cathode (right axis) and the corresponding variation in peak temperature from that calculated assuming emittances $=1$ (left axis) for various assumptions for true emittances. Data presented here were corrected using the nominal values shown as a solid line. excellent repeatability between scans. The uncertainty is dominated by three sources of systematic error. First among these is the $1 \%$ uncertainty in the thermocouple measurements used to calibrate the pyrometer response. This is presumably a systematic bias due to variations in the tungstenrhenium alloys or mechanical junctions of the thermocouples used in this experiment compared to those on which type $\mathrm{C}$ thermocouple tables are based. The error due to the difference between the orifice plate temperature and that of the downstream end of the insert during calibrations is reduced to $2-3{ }^{\circ} \mathrm{C}$ when the correction plotted in Fig. 8 is used. The uncertainty in the apparent emittance correction is bounded by considering the peak values and smoother tungsten values for the true emittances plotted in Fig. 10 compared to the nominal values. This source contributes up to +1 and -3 to $-9{ }^{\circ} \mathrm{C}$. These errors are assumed to be uncorrelated, and the total uncertainty given by the square root of the sum of the squares of these three contributors ranges from \pm 11 to $+12,-15{ }^{\circ} \mathrm{C}$ over a temperature range of $1025-1180{ }^{\circ} \mathrm{C}$.

\section{EXPERIMENTAL RESULTS}

\section{A. Operating conditions}

Data presented below were obtained after about $33 \mathrm{~h}$ of cumulative operation on a new cathode insert. Preliminary measurements with the probe and the thermocouples in the first few hours of operation indicated much higher temperatures $\left(80-100{ }^{\circ} \mathrm{C}\right)$. Large changes in temperatures measured with thermocouples on the exterior of the orifice plate have also been observed in extended duration tests. ${ }^{4,16}$ Data obtained here are representative of cathode operation after the large initial transient, but the cathode insert may not yet have reached true steady state conditions.

Measurements were obtained for discharge currents of 6-12 A and flow rates between the minimum flow without large voltage oscillations and 10 SCCM (SCCM denotes cubic centimeter per minute at STP). The calibration was repeated after every current level (every three to seven scans). We have observed that the measurements are repeatable at a given operating point to within $1-2{ }^{\circ} \mathrm{C}$, so, generally, only one scan was obtained at each setting to minimize fiber contamination. Some points were repeated to check long-term repeatability, as noted above. The data were analyzed using the calibration curve taken before that data set if the subsequent calibration indicated no major shifts in response.

\section{B. Cathode thermal behavior}

The temperature distribution measured at $12 \mathrm{~A}$ and 5.5 SCCM is shown in Fig. 11 with a polynomial fit. The residuals plotted above this curve show the noise typically observed for this temperature range. The standard deviation in these measurements is about $4.5^{\circ} \mathrm{C}$ and is dominated by a slight hysteresis. The curve fit is used to extrapolate $1.7 \mathrm{~mm}$ downstream to the temperature peak at the end of the insert. The temperatures measured with the thermocouples are also shown in Fig. 11. Under nearly isothermal conditions during calibrations, the thermocouples agreed to within $2{ }^{\circ} \mathrm{C}$ of one 


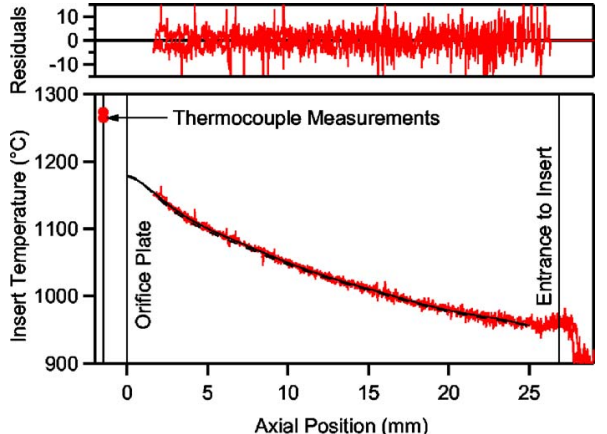

FIG. 11. Typical temperature distribution measurement and curve fit, showing extrapolation downstream to estimate peak temperature.

another. During cathode operation, however, they differed by $10-15^{\circ} \mathrm{C}$, indicating small variations in temperature near the tip.

Insert temperature distributions and peak orifice plate temperatures for currents of $6,8,10$, and $12 \mathrm{~A}$ are shown in Fig. 12. The flow rates for these measurements were 9, 8, 7, and 5.5 SCCM, respectively. As the flow rate was reduced to about 0.5 SCCM below these values, a sharp increase in the magnitude of the discharge voltage oscillations was observed. The minimum flow rate required for stable discharge operation depends on the electrode geometry and the strength of the applied magnetic field. The use of a flat plate anode downstream of the cathode and no applied field in these experiments led to a short residence time for primary electrons and relatively low gas pressure between the electrodes, compared to configurations with the anode enclosing the cathode and magnetic plasma confinement. High flow rates were therefore required for adequate plasma production in the interelectrode gap. The temperature drops monotonically from the downstream end of the insert and increases by approximately $25^{\circ} \mathrm{C}$ with every ampere increase in current. The peak insert temperatures are all less than the orifice plate temperatures.

A slight, but reproduceable, drop in the insert temperature with flow rate was observed at all current levels except the lowest, as shown in Fig. 13. In contrast, the orifice plate temperature increases slightly with increasing flow. The orifice plate temperatures displayed in this figure are the average of the two thermocouple readings and the error bars represent the $1 \%$ uncertainty in the thermocouple

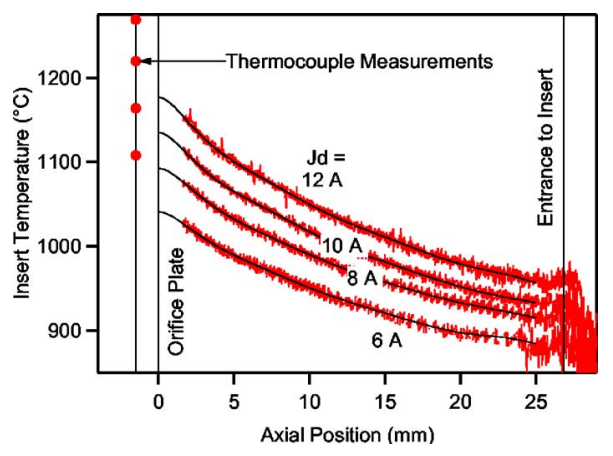

FIG. 12. Variation in temperature distribution with discharge current level for the minimum flow rates tested at each current; 9, 8, 7, and 5.5 SCCM for $6,8,10$, and $12 \mathrm{~A}$, respectively.

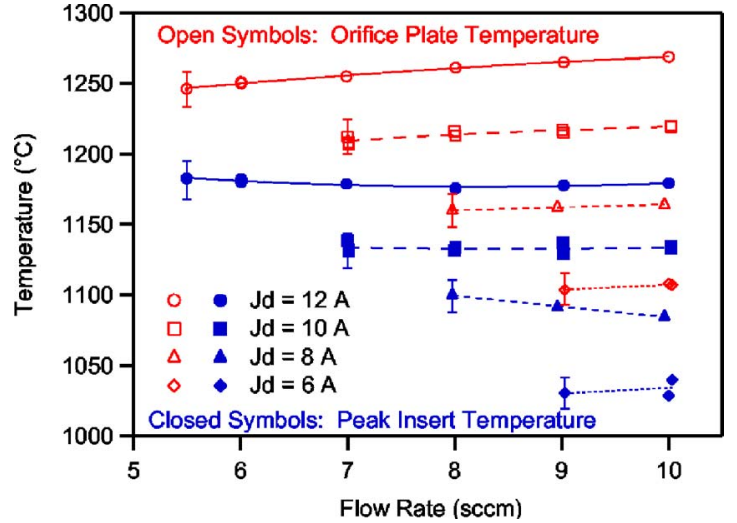

FIG. 13. Variation in peak insert and orifice plate temperatures with current and flow rate.

measurements. The insert temperature error bars were calculated using the methods described in Sec. III C.

The difference between the peak insert temperature and the orifice temperature for this range of currents and flow rates is plotted in Fig. 14. The difference varies between 60 and $90{ }^{\circ} \mathrm{C}$ and increases with both flow rate and current. Maximum and minimum uncertainties are shown as error bars on the 6 and 12 A curves. These do not include the $1 \%$ uncertainty in the thermocouple readings because this was assumed to systematically affect both measurements used to calculate the differences.

\section{DISCUSSION}

This data set represents the first comprehensive characterization of the temperature distribution on the actual emitting surface of an impregnated hollow cathode insert. The results demonstrate that the axial temperature profile increases monotonically along the insert by $170-200{ }^{\circ} \mathrm{C}$ with peak temperatures of $1025-1180{ }^{\circ} \mathrm{C}$ for discharge currents of 6-12 A. This is qualitatively similar to the behavior observed by Salhi et al. ${ }^{5}$ and Siegfried and Wilbur. ${ }^{8}$ The temperature increases significantly with discharge current (approximately $25^{\circ} \mathrm{C}$ for each ampere increase in current), but only shows a weak dependence on flow rate. This sensitivity to current level is similar to what Salhi et al. measured, but considerably less sensitive than the measurements of Siegfried and Wilbur (approximately $73{ }^{\circ} \mathrm{C} / \mathrm{A}$ ). The sensitivity to flow rate is undoubtedly due to variations in internal pres-

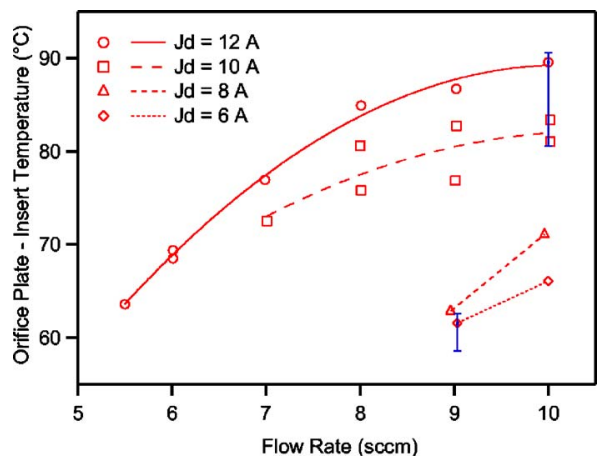

FIG. 14. Difference between peak insert and orifice plate temperatures as a function of current and flow rate. 
sure, similar to the effect observed by Siegfried and Wilbur. For the range of operating conditions and orifice geometry, the insert temperature was less than the orifice plate temperature by $60-90{ }^{\circ} \mathrm{C}$. The results indicate that the orifice plate temperature is determined largely by heat dissipation in the orifice, which increases with current and flow rate. The insert temperature for this cathode geometry is influenced by orifice plate heating as well as direct heat inputs from the plasma.

These measurements were enabled by the development of a robust, nonintrusive scanning fiber optic probe technique. Using rapid scans with a sapphire probe to overcome the high temperature plasma environment, narrow bandpass filters to eliminate line radiation, ratio pyrometry to minimize errors from source-detector distance variations, and in situ calibration and rigorous treatment of cavity emittance resulted in the resolution of very fine relative temperature changes. Uncertainties in the absolute temperature measurements ranged from \pm 11 to $+12,-15^{\circ} \mathrm{C}$ over the temperature range of $1025-1180{ }^{\circ} \mathrm{C}$. These uncertainties are dominated by a $1 \%$ uncertainty in the tungsten rhenium thermocouple measurements used in the calibration. Use of metal freezing point reference cavities for calibration could reduce this source of uncertainty to less than $1{ }^{\circ} \mathrm{C}$, yielding temperatures accurate to within a few degrees.

\section{ACKNOWLEDGMENTS}

The authors wish to thank Al Owens and Ray Swindlehurst for their assistance in the design and fabrication of the apparatus for this experiment. The research described in this article was carried out by the Jet Propulsion Laboratory, California Institute of Technology, under a contract with the National Aeronautics and Space Administration in support of Project Prometheus.

${ }^{1}$ J. E. Polk, J. R. Anderson, J. R. Brophy, V. K. Rawlin, M. J. Patterson, and J. S. Sovey, 35th Joint Propulsion Conference, Los Angeles, CA, 1999 (unpublished), Paper No. AIAA-99-2446.

${ }^{2}$ J. E. Polk et al., 27th International Electric Propulsion Conference, Pasadena, CA, 2001 (unpublished), Paper No. IEPC-01-075.

${ }^{3}$ A. Sengupta, 29th International Electric Propulsion Conference, Princeton, NJ, 2005 (unpublished), Paper No. IEPC-2005-026.

${ }^{4}$ T. R. Sarver-Verhey, 25th International Electric Propulsion Conference, Cleveland, OH, 1997 (unpublished), Paper No. IEPC 97-168.

${ }^{5}$ A. Salhi, R. M. Myers, and P. J. Turchi, 23rd International Electric Propulsion Conference, Seattle, WA, 1993 (unpublished), Paper No. AIAA93-025.

${ }^{6}$ T. R. Verhey, 21st International Electric Propulsion Conference, Orlando, FL, 1990 (unpublished), Paper No. AIAA-90-2586.

${ }^{7}$ S. D. Kovaleski and M. J. Patterson, 27th International Electric Propulsion Conference, Pasadena, CA, 2001 (unpublished), Paper No. IEPC-01-274.

${ }^{8}$ D. Siegfried and P. J. Wilbur, 14th International Electric Propulsion Conference, Princeton, NJ, 1979 (unpublished), Paper No. AIAA 79-2056.

${ }^{9}$ A. Salhi, Ph.D. thesis, The Ohio State University, Ohio, 1993.

${ }^{10}$ W. L. Ohlinger and K. M. Rebstock, 1994 Tri-Service/NASA Cathode Workshop, Cleveland, OH, 1994 (unpublished).

${ }^{11}$ T. R. Sarver-Verhey, 28th Joint Propulsion Conference, Nashville, TN, 1992 (unpublished), Paper No. AIAA-92-3204.

${ }^{12}$ J. P. Holman, Heat Transfer (McGraw Hill, New York, 1981).

${ }^{13}$ R. E. Rolling, A. I. Funai, and J. R. Grammer, USAF Report No. ML-TR64-363, 1964 (unpublished).

${ }^{14}$ D. F. Simmons, C. M. Fortgang, and D. B. Holtkamp, Report No. DOE/ NV/11718-572, 2001 (unpublished)

${ }^{15}$ G. Jaafara and B. Free, Appl. Surf. Sci. 24, 340 (1985).

${ }^{16}$ G. C. Soulas, 30th Joint Propulsion Conference, Indianapolis, 1994 (unpublished), Paper No. AIAA-94-3310. 